\title{
Mesenchymal Stem Cells-A Boon to Orthopedics
}

\author{
Ramesh R., Madhan Jeyaraman*, Kartavya Chaudhari, Hardik J. Dhamsania, Prajwal G. S. \\ Department of Orthopedics, JJM Medical College, Davangere, India \\ Email: ^madhanjeyarman@gmail.com
}

How to cite this paper: Ramesh R., Jeyaraman, M., Chaudhari, K., Dhamsania, H.J. and Prajwal G.S. (2018) Mesenchymal Stem Cells-A Boon to Orthopedics. Open Journal of Regenerative Medicine, 7, 19-27. https://doi.org/10.4236/ojrm.2018.72002

Received: May 17, 2018

Accepted: June 26, 2018

Published: June 29, 2018

Copyright ( $) 2018$ by authors and Scientific Research Publishing Inc. This work is licensed under the Creative Commons Attribution International License (CC BY 4.0).

http://creativecommons.org/licenses/by/4.0/ (c) (i) Open Access

\begin{abstract}
Percutaneous administration of "Orthobiologics" offers the advantage of accelerated and functional recovery of musculoskeletal disorders. Stem cell optimizes the biological healing of diseases. The regenerative capacity of mesenchymal stem cells provides a pavement for clinicians to build up an organ of interest in vitro. Mesenchymal stem cells remain the perfect cell based tissue regeneration modality for treatment of musculoskeletal disorders in a minimally invasive environment without any significant morbidity. This article outlines the applications of mesenchymal stem cell administration in various musculoskeletal disorders.
\end{abstract}

\section{Keywords}

Mesenchymal Stem Cells, Orthobiologics, Micromolecules, Differentiation, Growth Factors

\section{Introduction}

Percutaneous administration of "Orthobiologics" with osteoinductive and osteointegrative properties offers the advantage of accelerated and functional recovery of musculoskeletal disorders. Due to recent advances in molecular and tissue engineering and regeneration, the research on micromolecules has become the prime importance in the field of medical sciences. Stem cell offers a greater advantage of biological healing cascade without any significant morbidity to the individuals. The future relies on regenerative medicine called stem cells which has the scientific and ethical controversies. This article outlines the cell of interest called mesenchymal stem cells usage in orthopaedics.

\section{Orthobiologics}

"Orthobiologics" are the biological agents which help in healing the muscular and skeletal disorders within a micromolecular and natural healing environment. 
The bases of orthobiologics are:

- Matrix materials-Forms the ground substance and scaffold or frame work up-on which the stem cells forms the targeted tissue of interest.

- Growth factors-Potent substances to induce the tissue of interest.

- Stem cells-Biological cells which are cultured to produce the tissue of interest.

There are several forms of orthobiologics which are as follows:

1) Bone grafts.

2) Bone marrow aspirate.

3) Demineralised bone matrix.

4) Synthetic bone grafts.

5) Autologous blood injection.

6) Autologous conditioned serum.

7) Platelet rich plasma.

8) Stem cells.

9) Growth factors.

10) Bone morphogenetic proteins.

\section{Stem cells}

Stem cells are the naïve cells, blank cells (don't possess any cell surface markers) and uncommitted cells which can be differentiated into other cell types and can self renew to produce same type of stem cells exponentially in a controlled manner (Figure 1). Broadly stem cells are classified into:

a) Embryonic stem cells (ESC) [1] which are found in the inner mass of blastocyst,

b) Adult stem cells (ASC) [1] which are found in bone marrow, adipose tissue or blood cells,

c) Induced pluripotent stem cells (iPSC) [1] which are cultured in the laboratory to produce the tissue of interest.

Dynamics of stem cells [1]

Properties of stem cell [1]

A) Self renewal

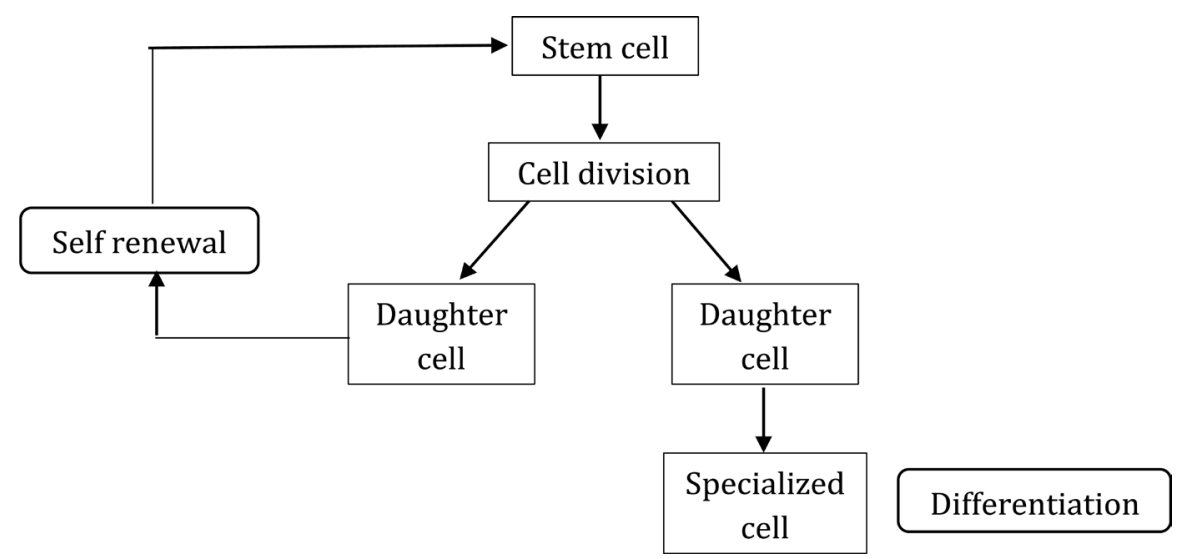

Figure 1. Dynamics of stem cells. 
- Obligatory asymmetric replication-Renewal of stem cell from a daughter cell.

- Stochastic differentiation-Differentiation of one daughter cell into stem cell and differentiation of one daughter cell to undergo mitosis to form another stem cell.

B) Differentiation

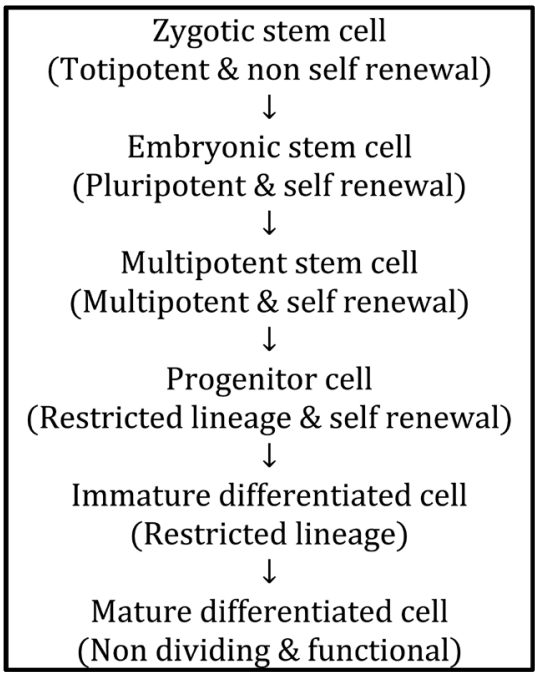

\section{Techniques of stem cell application}

a) Direct delivery-The aspirated bone or bone marrow is directly inserted to the injury site. This facilitates direct delivery of stem cells to the injury site and hastens the healing cascade.

b) Cell based delivery-Bone marrow is aspirated, and the stem cells are isolated, cultured and differentiated in laboratory media to increase their numbers before being applied to the injury site.

\section{Embryonic stem cells (ESC)}

Embryonic stem cells (ES cells or ESCs) are pluripotent stem cells derived from the inner cell mass of a blastocyst, an early stage pre-implantation embryo. Human embryos reach the blastocyst stage $4-5$ days post fertilization, at which time they consist of $50-150$ cells. Isolating the embryoblast, or inner cell mass (ICM) results in destruction of the blastocyst, a process which raises ethical issues.

Properties of embryonic stem cells

Embryonic stem cells are pluripotent which are able to differentiate to generate primitive ectoderm, which ultimately differentiates during gastrulation into all derivatives of the three primary germ layers: ectoderm, endoderm, and mesoderm. Under defined conditions, embryonic stem cells are capable of propagating indefinitely in an undifferentiated state. Conditions must either prevent the cells from clumping, or maintain an environment that supports an unspecialized state. When provided with the appropriate signals, to differentiate (presumably via the initial formation of precursor cells) into nearly all mature cell phenotypes. 
Uses of ESC are a) regenerative medicine and tissue replacement after injury or disease and b) pluripotent stem cells are used in the treatment of spinal cord injuries, age related macular degeneration, diabetes, neurodegenerative disorders (such as Parkinson's disease), AIDS.

\section{Adult stem cells (ASC)}

Adult stem cells, known as somatic stem cells, are undifferentiated cells, found throughout the body after development that multiply by cell division to replenish dying cells and regenerate damaged tissues. ASC have the inherent property of self renewal and multidifferentiative potential. The signalling pathways present in ASC are a) NOTCH gene pathway for hematopoietic, neural and mammary stem cells, b) Wnt signalling pathway for proposed stem cell regulators and c) TGF- $\beta$ to regulate the stemness of both normal and cancer stem cells.

Types of adult stem cells:

a) Hematopoietic stem cell-Bone marrow and umbilical cord.

b) Mammary stem cell-Luminal and myoepithelial cell types of the gland.

c) Intestinal stem cells-Crypts of Lieberkuhn.

d) Mesenchymal stem cells.

e) Endothelial stem cell-Cells that line blood vessels.

f) Neural stem cell-Present in the subventricular zone, which lines the lateral ventricles, and the dentate gyrus of the hippocampal formation.

g) Olfactory adult stem cells.

h) Ocular stem cells-Found in the area of limbus.

i) Neural crest stem cells-Hair follicles which represents a remnant of stem cells of embryonic neural crest. These cells can generate neurons, Schwann cells, myofibroblast, chondrocytes and melanocytes.

j) Testicular stem cells-Spermatogonial progenitor cells found in the testicles of laboratory mice by scientists in Germany and the United States.

\section{Induced pluripotent stem cells}

Induced pluripotent stem cells (also known as iPS cells or iPSCs) are a type of pluripotent stem cell that can be generated directly from adult cells. iPSCs are typically derived by introducing products of specific sets of pluripotency-associated genes, or "reprogramming factors", into a given cell type. iPSC derivation is typically a slow and inefficient process, taking 1 - 2 weeks for mouse cells and 3 - 4 weeks for human cells, with efficiencies around $0.01 \%-0.1 \%$. Upon introduction of reprogramming factors, cells begin to form colonies that resemble pluripotent stem cells, which can be isolated based on their morphology, conditions that select for their growth, or through expression of surface markers or reporter genes. The challenges in iPSC are low efficiency, inadequate programming, genomic insertion and tumorigenic potential.

Method of induction of cells:

- Isolate and culture donor cells.

- Transduce stem cell-associated genes into the cells by viral vectors. Red cells indicate the cells expressing the exogenous genes. 
- Harvest and culture the cells according to ES cell culture, using mitotically inactivated feeder cells.

- A small subset of the transfected cells become iPS cells and generate ES-like colonies.

\section{Mesenchymal stem cells (MSCs)}

Mesenchymal stem cells are the specialized form of adult stem cells which has the potential to differentiate into multi-lineage cell lines (Figure 2) such as osteocyte, chondrocyte, adipocyte, neurocyte, myocyte, histiocyte and stromal cells. MSCs are found in bone marrow, cord cells, adipose tissue, amniotic fluid and molar teeth.

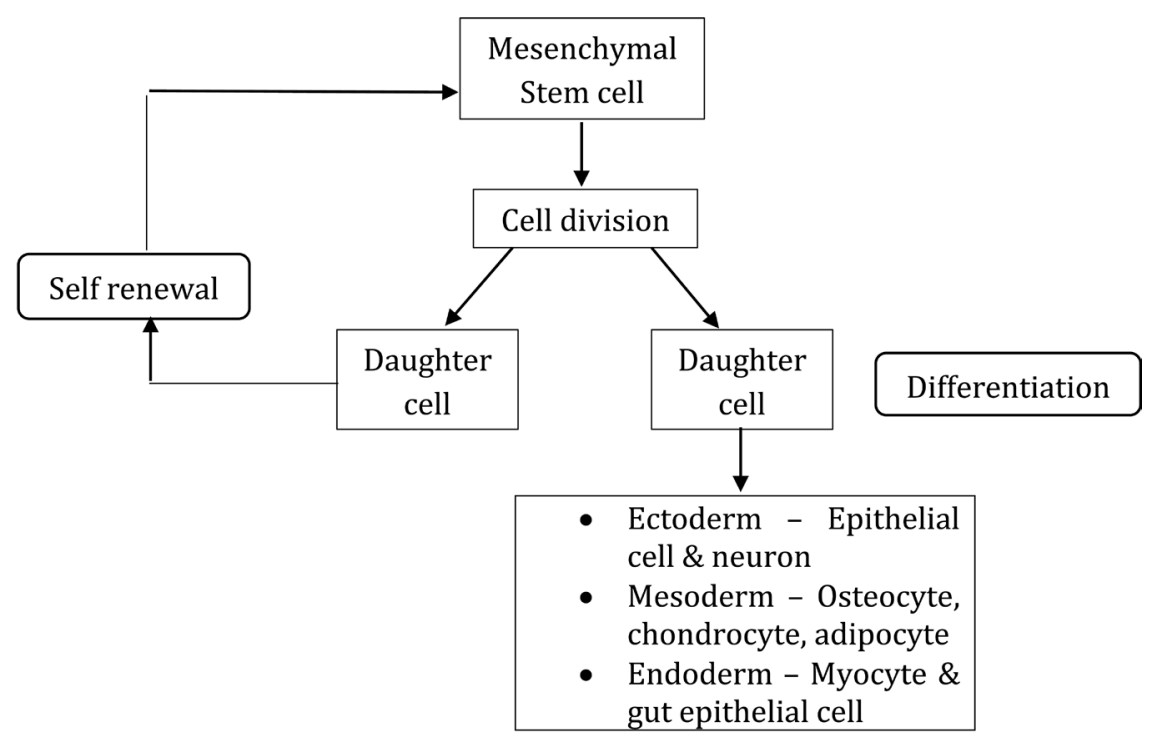

Figure 2. Biology of mesenchymal stem cells.

\section{Salient features of MSC [2] [3]}

- Adherent fibroblast like morphology.

- No expression of hematopoietic stem cell markers such as CD34 and CD14.

- Expression of matrix markers such as CD44, CD29 and CD71.

- Expression of mesenchymal stem cell markers, SH2 and SH105.

- Contains extra cellular matrix components such as collagen, proteoglycans and fibronectin.

- Growth factors and cytokine receptors for TGF- $\beta$.

- Anti proliferative activity to inhibit T cell proliferation into tumorigenic cells.

- Favours immunoprivilege \& allogenic preparation.

\section{Culture of MSC}

Mesenchymal stem cells are cultured in basal medium such as Dulbecco's modified Eagle's medium (high glucose) in the presence of $10 \%$ fetal bovine serum (FBS). In culture, MSCs have a fibroblastic morphology. Primary cultures are usually maintained for 12 - 16 days, during which haematopoietic cell fraction is depleted. The addition of growth factor supplements such as fibroblast growth factor-2 (FGF-2) to primary cultures of human MSCs to lead to an en- 
hanced osteogenic potential [2] [3].

\section{Immune modulation of MSCs [4]}

\section{a) T lymphocyte system}

MSC-mediated immunoregulation is mediated through nitric oxide and indoleamine 2,3-dioxygenase (IDO), which are only released by MSCs after triggering by IFN $\gamma$ produced by target cells. IDO inhibits the proliferation of IFN $\gamma$-producing TH1 cells and, with $\mathrm{PGE}_{2}$, to block NK-cell activity.

\section{b) HLA-G5 system}

MSCs produce soluble HLA-G5 which suppress T-cell proliferation, NK-cell and $\mathrm{T}$-cell cytotoxicity, and promotes regulatory $\mathrm{T}$ cell production.

\section{Application of MHCs in Orthopedics}

\section{a) Cartilage}

Cartilage is an avascular and aneural structure which has very limited potential for regeneration. Mesenchymal stem cells are used to provide a scaffold and meshwork to rejuvenate the focal chondral defect by autologous chondrocyte implantation (ACI). ACI is a two-stage procedure, with assessment of the chondral injury and biopsy to harvest 200 to $300 \mathrm{mg}$ of healthy cartilage, followed by an enzymatic digestion and cell expansion in monolayer culture with cryopreservation of the cells which is further thawed and expanded to the target cell population. During the second stage, a biological reconstruction is performed to repair the articular cartilage defects to hasten the recovery with a successful functional outcome [5].

For osteoarthritis knee, the delivery of ultrasonicated adipose tissue offers a great advantage in proliferation of chondrocytes along with growth factors leads to better pain relief and functional improvement in quality of life. The dose of adipose tissue to be injected depends upon the grade of osteoarthritis and BMI of the individual [6].

b) Bone

$\mathrm{BMP}$ receptor $1 \mathrm{~B}$ is required for mesenchymal stem cell to form osteoblastic lineage in bone defects. Mesenchymal stem cells are used to enhance the regeneration of osseous defect in case of segmental bone loss, established non union and regeneration of physeal defect in children [7] [8]. The optimal loading of MSC on-to a carrier such as retroviral or adenoviral molecule offers a great advantage in minimally invasive procedure than conventional open surgical techniques. These MSC should possess the properties of osteoinduction, osteoconduction, osteointegration and mechanical stimulation to regenerate osseous defects. In segmental bone loss, calcium carbonate or hydroxyapatite scaffold is inserted upon which MSC are delivered to promote osteogenesis and osteointegration [9].

c) Tendons

The chronic and overuse of tendons leads to degenerative conditions such as plantar fasciitis, interscapular fibrofasciitis, tennis elbow, golfer's elbow and tendinopathies. Such degenerated tendon has an inferior potential to regenerate. 
Autologous tenocyte injection is performed by a needle biopsy of healthy tendon which is subjected for isolation of cell of interest called tenocyte. Then tenocyte is amplified in cell cultured media and are injected into the injured or degenerated tendon [10].

Tendon bioengineering using mesenchymal stem cells and a silk scaffold, allogenic adipose tissue derived mesenchymal stem cells and injection of microRNA with a loss of miR29 which results in increase in the production of type collagen 3 [11].

d) Ligaments

Sports injuries will result in concomitant injury to ligaments which has very poor potential to regenerate. MSC along with bone morphogenetic protein 2 and 7 \& growth factors enhance neovascularization and regeneration of injured or degenerated ligaments. In ACL and meniscal rupture, intra-articular injection of MSCs with BMP $2 \& 7$ offers a biological healing of the ruptured ligaments. The scaffold based tissue bioengineering offers a greater advantage in ligamentous healing [12] [13].

e) Spine

MSC transplantation into the degenerated disc offers a potential to increase proteoglycans and glycosaminoglycans and induce degenerated spine \& disc for regeneration and prevents vertebral collapse. The transported MSC maintain the viability of cells and involve in synthesizing micromolecules responsible for maintenance of disc shape [14].

MSC has the potent ability to form neural tissue and offers a functional life to patients with traumatic paraplegia. The injection of MSC along with carrier molecule to the injured site allows optimized enhancement of remyelination of the neural tubes and axons thereby restoring the spinal reflex arcs [15].

f) Miscellaneous disorders

MSC has been tried in osteogenesis imperfecta where the defect lies in type 1 collagen synthesis. MSC are directed to increase the bone strength \& integrity and produces type 1 collagen which prevents recurrent fractures with trivial trauma. MSC eliminates the need of immunosuppression in patients with osteogenesis imperfecta [16].

In Duchene muscular dystrophy, the defect is due to mutation of dystrophin gene leads to the absence of dystrophin protein. MSC administration offers a greater advantage of contractile myotubules differentiation and regeneration of damaged myotubular system [17].

In avascular necrosis of bone, the primary pathology is idiopathic. Some theories states avascular necrosis is due to increased intra-osseous pressure. The aim of surgery is to decompress the intra-osseous pressure by drilling multiple holes in the bone. Delivering of MSCs in the drill holes leads to osteointegration and osteogenesis which helps in remodelling of the bony structures [18].

\section{Significance of MSC over ESC and iPSC}

- Mesenchymal stem cells are more advantageous than embryonic stem cells 
and induced pluripotent stem cells.

- MSC are easily accessible from the donor and to be cultured upon the laboratory.

- MSC does not raise any ethical or legal concerns when compared to embryonic stem cells.

- MSC does not possess any tumorigenic potential when compared to induced pluripotent stem cells.

- MSC possess immunoprivilege and immunomodulation at every point of cell cycle by which it is propagated to produce the tissue of interest.

\section{Conclusion}

MSCs admixed with micromolecules such as growth factors, cytokines \& BMPs provide a three dimensional structure and biomechanically, structurally and functionally compliant to the naïve cells of host origin. Further research on the dosage and protocols on MSC for various disorders should be formulated which leads to decreased morbidity and improve the functional quality of life.

\section{Conflicts of Interest}

The authors declare no conflicts of interest regarding the publication of this paper.

\section{References}

[1] Barry, F.P. and Murphy J.M. (2004) Mesenchymal Stem Cells: Clinical Applications and Biological Characterization The International Journal of Biochemistry \& Cell Biology, 36, 568-584. https://doi.org/10.1016/j.biocel.2003.11.001

[2] Deans, R.J. and Moseley, A.B. (2000) Mesenchymal Stem Cells. Experimental Hematology, 28, 875-884. https://doi.org/10.1016/S0301-472X(00)00482-3

[3] Pittenger, M.F., Flake, A.M. and Deans, R.J. (2002) Stem Cell Culture: Mesenchymal Stem Cells from Bone Marrow. In: Atala, A. and Lanza, R.P., Eds., Methods of Tissue Engineering, Academic Press, San Diego, 461-469.

[4] Uccelli, A., Moretta, L. and Pistoia, V. (2008) Mesenchymal Stem Cells in Health and Disease. Nature Reviews Immunology, 8, 726-736. https://doi.org/10.1038/nri2395

[5] Their, S., Weiss, C. and Fickert, S. (2017) Arthroscopic Autologous Chondrocyte Implantation in the Hip for the Treatment of Full-Thickness Cartilage Defects: A Case Series of 29 Patients and Review of the Literature. SICOT-J, 3, 72 https://doi.org/10.1051/sicotj/2017037

[6] Wakitani, S., Goto, T., Pineda, S.J., et al. (1994) Mesenchymal Cell-Based Repair of Large, Full Thickness Defects of Articular Cartilage. The Journal of Bone and Joint Surgery, 76, 579-592. https://doi.org/10.2106/00004623-199404000-00013

[7] Jaiswal, N., Haynesworth, S.E., Caplan, A.I. and Bruder, S.P. (1997) Osteogenic Differentiation of Purified, Culture-Expanded Human Mesenchymal Stem Cells in $\mathrm{Vi}$ tro. Journal of Cellular Biochemistry, 64, 295-312. https://doi.org/10.1002/(SICI)1097-4644(199702)64:2<295::AID-JCB12>3.0.CO;2-I

[8] Reddi, A.H. (1998) Initiation of Fracture Repair by Bone Morphogenetic Proteins. 
Clinical Orthopaedics and Related Research, 355, 66-72. https://doi.org/10.1097/00003086-199810001-00008

[9] Ahn, J.I., Terry Canale, S., Butler, S.D. and Hasty, K.A. (2004) Stem Cell Repair of Physeal Cartilage. Journal of Orthopaedic Research, 22, 1215-1221.

https://doi.org/10.1016/j.orthres.2004.03.012

[10] Wang, A.W., Bauer, S., Goonatillake, M., Breidahl, W. and Zheng, M.-H. (2013) Autologous Tenocyte Implantation, a Novel Treatment for Partial Thickness Rotator Cuff Tear and Tendinopathy in an Elite Athelete. BMJ Case Reports, 2013. https://doi.org/10.1136/bcr-2012-007899

[11] Rodeo, S.A., Suzuki, K., Deng, X.H., Wozney, J. and Warren, R.F. (1999) Use of Recombinant Human Bone Morphogenetic Protein-2 to Enhance Tendon Healing in a Bone Tunnel. The American Journal of Sports Medicine, 27, 476-488. https://doi.org/10.1177/03635465990270041201

[12] Hyman, J. and Rodeo, S.A. (2000) Injury and Repair of Tendons and Ligaments. Physical Medicine and Rehabilitation Clinics of North America, 11, 267-288. https://doi.org/10.1016/S1047-9651(18)30129-3

[13] Peretti, G.M., Caruso, E.M., Randolph, M.A. and Zaleske, D.J. (2001) Meniscal Repair Using Engineered Tissue. Journal of Orthopaedic Research, 19, 278-285. https://doi.org/10.1016/S0736-0266(00)90010-X

[14] Crevensten, G., Walsh, A.J., Ananthakrishnan, D., et al. (2004) Intervertebral Disc Cell Therapy for Regeneration: Mesenchymal Stem Cell Implantation in Rat Intervertebral Discs. Annals of Biomedical Engineering, 32, 430-434. https://doi.org/10.1023/B:ABME.0000017545.84833.7c

[15] Akiyama, Y., Radtke, C., Honmou, O. and Kocsis, J.D. (2002) Remyelination of the Spinal Cord Following Intravenous Delivery of Bone Marrow Cells. Glia, 39, 229-236. https://doi.org/10.1002/glia.10102

[16] Horwitz, E.M., Prockop, D.J., Fitzpatrick, L.A., et al. (1999) Transplantability and Therapeutic Effects of Bone Marrow-Derived Mesenchymal Cells in Children with Osteogenesis Imperfecta. Nature Medicine, 5, 309-313. https://doi.org/10.1038/6529

[17] Gussoni, E., Soneoka, Y., Strickland, C.D., et al. (1999) Dystrophin Expression in the MDX Mouse Restored by Stem Cell Transplantation. Nature, 401, 390-394. https://doi.org/10.1038/43919

[18] Houdek, M.T., Wyles, C.C., Martin, J.R. and Siera, R.J. (2014) Stem Cell Treatment for Avascular Necrosis of the Femoral Head: Current Perspectives. Stem Cell Cloning, 7, 65-70. 